\title{
Association between maternal adherence to healthy lifestyle practices and risk of obesity in offspring: results from two pro- spective cohort studies of mother-child pairs in the United States
}

\author{
Klodian Dhana, ${ }^{1}$ Jess Haines, ${ }^{2}$ Gang Liu, ${ }^{1}$ Cuilin Zhang, ${ }^{3}$ Xiaobin Wang, ${ }^{4}$ Alison E Field, ${ }^{5}$ \\ Jorge E Chavarro, ${ }^{1,6}$ Qi Sun ${ }^{1,6}$
}

${ }^{1}$ Department of Nutrition,

Harvard TH Chan School of

Public Health, Boston, MA

02115, USA

${ }^{2}$ Department of Family Relations and Applied Nutrition at the

University of Guelph in Guelph,

Canada

${ }^{3}$ Division of Intramural

Population Health Research,

Eunice Kennedy Shriver

National Institute of

Child Health and Human

Development, National

Institutes of Health, Bethesda,

MD, USA

${ }^{4}$ Center on the Early Life

Origins of Disease, Department

of Population, Family and

Reproductive Health, Johns

Hopkins Bloomberg School of

Public Health, Baltimore, MD,

USA

${ }^{5}$ Department of Epidemiology,

Brown University School of

Public Health, Providence, RI,

USA

${ }^{6}$ Channing Division of Network

Medicine, Department of

Medicine, Brigham and

Women's Hospital, and Harvard

Medical School, Boston, MA,

USA

Correspondence to:

Q Sun qisun@hsph.harvard.edu

Additional material is published online only. To view please visit the journal online.

Cite this as: BMJ 2018;362:k2486 http://dx.doi.org/10.1136/bmj.k2486

Accepted: 8 May 2018

\begin{abstract}
Objective

To examine the association between an overall maternal healthy lifestyle (characterized by a healthy body mass index, high quality diet, regular exercise, no smoking, and light to moderate alcohol intake) and the risk of developing obesity in offspring.
\end{abstract}

Design

Prospective cohort studies of mother-child pairs.

Setting

Nurses' Health Study II (NHSII) and Growing Up Today Study (GUTS) in the United States.

Participants

24289 GUTS participants aged 9-14 years at baseline who were free of obesity and born to $16945 \mathrm{NHSII}$ women.

\section{Main outcome measure}

Obesity in childhood and adolescence, defined by age and sex specific cutoff points from the International Obesity Task Force. Risk of offspring obesity was evaluated by multivariable log-binomial regression models with generalized estimating equations and an exchangeable correlation structure.

\section{Results}

1282 (5.3\%) offspring became obese during a median of five years of follow-up. Risk of incident obesity was lower among offspring whose mothers maintained a healthy body mass index of 18.5-24.9 (relative risk $0.44,95 \%$ confidence interval 0.39 to 0.50 ), engaged in at least $150 \mathrm{~min} /$ week of moderate/ vigorous physical activities $(0.79,0.69$ to 0.91$)$, did not smoke $(0.69,0.56$ to 0.86$)$, and consumed alcohol in moderation (1.0-14.9 g/day; $0.88,0.79$ to 0.99), compared with the rest. Maternal high quality

\section{WHAT IS ALREADY KNOWN ON THIS TOPIC}

In women, adhering to an overall healthy lifestyle is associated with a substantially reduced risk of type 2 diabetes, coronary heart disease, and mortality

Whether such a healthy lifestyle exerts health effects among offspring, possibly through modulating the living environment and lifestyle of children, deserves examination

\section{WHAT THIS STUDY ADDS}

Offspring of women adhering to an overall healthy lifestyle had a substantially lower risk of obesity than children of mothers who did not practice these lifestyle choices

These findings highlight the potential benefits of implementing parent based multifactorial interventions to curb the risk of childhood obesity

diet (top $40 \%$ of the Alternate Healthy Eating Index 2010 diet score) was not significantly associated with the risk of obesity in offspring $(0.97,0.83$ to 1.12$)$. When all healthy lifestyle factors were considered simultaneously, offspring of women who adhered to all five low risk lifestyle factors had a $75 \%$ lower risk of obesity than offspring of mothers who did not adhere to any low risk factor $(0.25,0.14$ to 0.47$)$. This association was similar across sex and age groups and persisted in subgroups of children with various risk profiles defined by factors such as pregnancy complications, birth weight, gestational age, and gestational weight gain. Children's lifestyle did not significantly account for the association between maternal lifestyle and offspring obesity risk, but when both mothers and offspring adhered to a healthy lifestyle, the risk of developing obesity fell further $(0.18,0.09$ to 0.37$)$.

\section{Conclusion}

Our study indicates that adherence to a healthy lifestyle in mothers during their offspring's childhood and adolescence is associated with a substantially reduced risk of obesity in the children. These findings highlight the potential benefits of implementing family or parental based multifactorial interventions to curb the risk of childhood obesity.

\section{Introduction}

One in five American children and adolescents aged 6-19 years is obese. ${ }^{1}$ Obesity in childhood is associated with an increased risk of multiple metabolic disorders, ${ }^{2}$ including diabetes and cardiovascular disease, as well as premature death, in adulthood. ${ }^{34}$ Identifying modifiable risk factors for the prevention of childhood obesity has become a public health priority.

While the role of genetic underpinning of obesity is widely recognized, ${ }^{5}$ a rapid increase in obesity in recent years is more likely to be attributable to changes in lifestyle, ${ }^{6-8}$ suggesting that "nurture" carries more weight than "nature" in driving the current pandemic of obesity. ${ }^{9}$ Lifestyle factors contributing to childhood obesity include lack of physical activity, sedentary activities, and eating a high calorie diet among children. $^{10-12}$ Studies have shown that children's lifestyle choices are largely influenced by their mothers. ${ }^{13-16}$ In addition, maternal behaviors such as smoking and alcohol consumption are also associated with offspring's body mass index. ${ }^{17-19}$ Overall, these lines of evidence imply that maternal lifestyle choices could exert health effects among offspring, probably through modulating the living environment and lifestyle of children. 
Despite the fact that these lifestyle choices are often interconnected with each other, no study has yet examined the effect of an overall healthy maternal lifestyle characterized by consumption of a healthy diet, maintaining a healthy weight, regular physical activity, light to moderate alcohol consumption, and no smoking that jointly could have a greater influence on children's environment and lifestyle than any individual factors. ${ }^{20}$ Solid evidence has suggested that adherence to such a healthy lifestyle is associated with a substantially lower risk of morbidity and mortality in adult women. ${ }^{21-23}$ However, it is unknown whether healthy lifestyle patterns in mothers during their offspring's childhood and adolescence influence the development of obesity in their children, potentially through modulating the environment in which children grow and develop before adulthood.

Therefore, we aimed to determine prospectively the association of overall maternal lifestyle during offspring's childhood and adolescence with the risk of incident obesity during age 9-18 years, using data from mother-child pairs enrolled in the Nurses' Health Study II and Growing Up Today Study. We also examined joint associations between maternal and offspring lifestyle factors and childhood obesity risk.

\section{Methods}

Study population

Nurses' Health Study II (NHSII) and Growing Up Today Study (GUTS)

NHSII is an ongoing prospective cohort study established in 1989 with the recruitment of 116430 female nurses aged 25-42 years who responded to a detailed questionnaire about their lifestyle characteristics and medical history at baseline, and this information was updated every two years. ${ }^{24}$ Dietary data were first collected in 1991 and were updated every four years thereafter by use of a validated food frequency questionnaire.

In 1996, participants of NHSII who had children aged 9-14 years received an invitation letter for enrolling their children in the GUTS cohort. A total of 16882 children returned completed questionnaires. In 2004, GUTS was extended to include 10918 more children of NHSII participants, who were aged 9-14 years. GUTS participants have been followed up with yearly self administered follow-up questionnaires between 1997 and 2001 and with biennial questionnaires thereafter up to 2013. We excluded NHSII participants who were pregnant during followup; had missing data of body mass index, physical activity, or smoking status; or left blank more than 10 items of the food frequency questionnaire during follow-up. We also excluded GUTS participants who did not report their weight and height at baseline or were obese at the entry of study. The final study included 24289 GUTS participants born to 16945 NHSII women at baseline.

The study was approved by the human subjects committees of the Harvard T H Chan School of Public Health and Brigham and Women's Hospital. In
NHSII and GUTS, the return of the questionnaire was considered as informed consent.

\section{Assessment of lifestyle factors in mothers}

Diet was assessed by use of a 130 item food frequency questionnaire, which was designed to measure diet over the past year. ${ }^{25} 26$ Participants were asked how often, on average, they had consumed specific foods, with nine possible frequencies ranging from "never" to "at least six times per day" of prespecified portion sizes. To assess the overall diet quality, we calculated the Alternate Healthy Eating Index 2010 diet score, ${ }^{27}$ which summarizes information on the following dietary factors:

- Higher intakes of vegetables

- Fruits

- Nuts

- Whole grains

- Polyunsaturated fatty acids

- Long chain omega 3 fatty acids

- Lower intakes of red and processed meats, sugar sweetened beverages, trans fats, and sodium.

Information on alcohol consumption was obtained from food frequency questionnaires, and participants reported their average frequency of alcoholic beverage intake during the previous year. ${ }^{25}$ Physical activity was assessed by a validated questionnaire, ${ }^{28}$ which inquired about the average time per week participants spent in any of the moderate or vigorous activities during the preceding year. Participants reported body height and weight at baseline and updated body weight in the biennial questionnaires. We computed mothers' body mass index by dividing their weight $(\mathrm{kg})$ by height squared $\left(\mathrm{m}^{2}\right)$. NHSII participants were inquired about their smoking history, including current smoking status, the number of cigarettes smoked per day for current smokers, years of quitting for past smokers, and other related information at the biennial questionnaires.

\section{Definition of low risk group}

Based on the evidence for health benefits of lifestyle factors in the prevention of chronic diseases and mortality among women, ${ }^{21-23}$ we considered, a priori, five low risk lifestyle factors. These factors included an Alternate Healthy Eating Index 2010 diet score in the top $40 \%$, body mass index $18.5-24.9$, abstinence from cigarette smoking, light to moderate consumption of alcohol (1.0-14.9 g/day), and engaging in physical activity for at least 150 minutes per week with moderate or vigorous intensity. For each low risk factor, the NHSII participants received a score of 1 or otherwise 0 . The sum of these five scores gave a final score within the range of 0-5, with higher scores indicating a healthier lifestyle.

\section{Assessment of covariates and potential effect modifiers}

Covariates considered in the analysis included maternal factors (such as age at baseline, ethnicity, 
history chronic diseases, living status, household income, and educational attainment of spouse/ partner) and offspring factors (including sex, diet, eating behaviors, physical activity, sedentary time, and calorie intake). Maternal and offspring factors were gathered from NHSII and GUTS follow-up questionnaires, respectively. NHSII participants reported in the 2009 questionnaire their gestational age, birth weight, type of delivery, and pregnancy complications for their offspring included in the study. The GUTS participants self reported their height, weight, diet, physical activity, and eating behaviors at follow-up questionnaires. In GUTS, diet was assessed by the Youth/Adolescent Questionnaire at each wave of GUTS follow-up, ${ }^{29}$ and we also used the Alternate Healthy Eating Index 2010 diet score to estimate the quality of diet in children. Eating behaviors in offspring were evaluated by frequencies of breakfast consumption, and whether they consumed preprepared dinners or fried foods at home and elsewhere. GUTS participants reported how many hours per week and in each season they engaged in physical activity over the past year. Based on this information, we calculated hours per week each participant spent on physical activities. Similarly, we estimated sedentary behavior by calculating total hours per week participants spent over the past year watching television, using the computer, surfing the internet, and reading or doing homework.

\section{Outcome assessment}

Body mass index in offspring was calculated by use of self reported weight and height. Previous studies have found that self reported and measured weight and height are reasonably correlated in children and adolescents. $^{30-33}$ To define obesity in childhood and adolescence, we used the age and sex specific cutoff values of body mass index from the International Obesity Task Force. ${ }^{34}$ Our outcome was the incident onset of obesity during follow-up in GUTS.

About $16.5 \% \quad(n=4008)$ of GUTS participants were lost to follow-up, which was defined as a GUTS participant not returning any questionnaire during the last three calendar years of follow-up. To investigate whether the probability of dropout was independent of maternal lifestyle factors, we computed a propensity score of dropout using a logistic regression. In this analysis, dropout from the study (yes/no) was treated as the dependent variable, and low risk maternal lifestyle score ( 0 to 5 low risk factors) was the independent variable. We computed the distribution of the estimated propensity score for offspring who dropped out and those who remained in the study (supplementary figure 1). The results demonstrated that the probability of dropout was independent of maternal lifestyle.

Among the GUTS participants, we imputed missing body mass index during the follow-up using a multiple imputation approach (SAS PROC MI procedure). The predictor variables included the offspring's valid body mass index assessments at baseline and during follow- up, as well as age, sex, diet, physical activity, sedentary time, and calorie intake at baseline and during followup. We evaluated the validity of imputations in our study by randomly assigning missing values to valid body mass index values, imputing the missing values, and then estimating misclassification of obesity status by imputed values. In short, we randomly assigned missing values among $10 \% \quad(n=1821)$ of GUTS participants who had at least three valid assessments of body mass index $(n=18585)$. We chose offspring with at least three valid assessments of body mass index because $90 \%$ ( $n=22$ 008) of offspring in our study had at least two valid assessments. For this analysis for demonstrating validity, we followed the same method described above (SAS PROC MI procedure) to impute missing values.

After imputation, we calculated the age and sex specific obesity status based on the valid and imputed body mass index values, respectively, and evaluated misclassification by imputed values. We found that the vast majority of individuals could be correctly classified regarding obesity status on the basis of imputed body mass index: $97.4 \%(n=1773)$ of individuals were correctly classified. However, the imputed values more accurately classified nonobese status than obese status: the misclassification was $0.7 \%$ (that is, specificity $99.3 \%$ ) and $38.7 \%$ (that is, sensitivity 61.3\%), respectively. To further visualize the misclassification of body mass index, we constructed a Bland-Altman agreement plot, ${ }^{35}$ where the difference of the imputed and valid measures were plotted against the mean of imputed and valid body mass index (supplementary figure 2).

\section{Statistical analysis}

The follow-up period of the study was from the baseline (1995 for NHSII; and 1996 for GUTS) until one of the following events, whichever came first: onset of obesity in the offspring; when the offspring reached the age of 18 years, after which offspring might not continue to live with the mother; or the end of follow-up. For each continuous lifestyle variable of the mother (that is, body mass index, diet, physical activity, and alcohol intake), we calculated the cumulative average starting from the baseline until the onset of offspring obesity or the end of follow-up. Similarly, we calculated the cumulative averages for continuous lifestyle variables of the offspring (that is, diet, physical activity, sedentary time, and calorie intake). For categorical variables, including smoking status in mothers and eating behaviors in offspring, we used the most recent available information before the onset of obesity in offspring or the last measurement before the end of follow-up.

To evaluate the association between maternal lifestyle factors and offspring obesity, we calculated relative risks and 95\% confidence intervals using multivariable log-binomial regression models with generalized estimating equations and specified an exchangeable correlation structure. Correlations of outcomes between siblings born to the same 
mother were accounted for by use of the generalized estimating equations model. We first evaluated associations with offspring obesity by categories of each low risk factor, adjusting for other maternal factors, including age at baseline, race or ethnicity, history of chronic diseases, living status, household income in 2001, and educational attainment of spouse or partner, as well as offspring sex. We then derived a low risk lifestyle score by summing the number of low risk lifestyle factors. We began by considering diet, physical activity, and alcohol intake in the score. We then added smoking status, and, finally, body mass index to the score to examine all five factors simultaneously. In those analyses, we compared the characteristics of offspring born to mothers who adhered to all low risk factors at issue with other offspring of women who did not adhere to the low risk factors.

We generated a missing category for maternal covariates, such as household income $(n=3233$, $19.1 \%$ ) and educational attainment of spouse or partner ( $\mathrm{n}=1340,7.9 \%)$. Missing values of offspring covariates including diet, physical activity, and sedentary time were also imputed by the multiple imputation approach mentioned above. PROC MIANALYZE was used to calculate the composite relative risks and $95 \%$ confidence intervals and to generate valid statistical inferences from five imputed datasets.

Moreover, we explored the role of offspring lifestyle in the associations between maternal lifestyle and the risk of obesity in offspring. We first examined correlations between maternal and offspring lifestyle factors by Spearman correlation coefficients. We further evaluated whether children's behaviors, including diet, eating behaviors, physical activity, sedentary time, and calorie intake might mediate the associations of maternal lifestyle factors during offspring childhood and adolescence with the risk of obesity in offspring. Lastly, we estimated the risk of obesity in offspring according to the combination of maternal lifestyle and offspring lifestyle.

In secondary analyses, we evaluated effect modification by risk factors of offspring obesity, such as pregnancy complications, gestational age, gestational weight gain, and maternal body mass index. Sex and age specific associations were examined by conducting analyses in boys and girls and in offspring aged 9-11 and 12-14 years, respectively. We repeated the analyses among NHSII participants without missing covariate data, as well as among offspring with valid assessments of body mass index only. We also evaluated the associations after excluding former smokers from the low risk group. Finally, we assessed whether prepregnancy maternal lifestyle accounted for the association between maternal lifestyle during offspring childhood and adolescence and the risk of offspring obesity. All statistical tests were two sided and were considered statistically significant at $\mathrm{P}<0.05$. All analyses were conducted by SAS version 9.4 (SAS Institute).

\section{Patient involvement}

No patients were involved in setting the research question or the outcome measures, nor were they involved in the design and implementation of the study. There are no plans to involve patients in dissemination.

\section{Results}

Table 1 presents the characteristics of the 16945 mothers (NHSII) and their 24289 offspring (GUTS) included in our analysis. At baseline, women were, on average, 41 years old, had a mean body mass index of 25.1, and most ( $n=15807,93.3 \%)$ were not current smokers. Their offspring were, on average, 12 years old, and $45.8 \%$ ( $n=11133)$ were boys.

Table 2 shows the associations between individual maternal lifestyle factors and risk of offspring obesity. We identified 1282 (5.3\%) offspring who became obese during a median of five years of follow-up. In multivariable analyses (model 2), offspring of women with obesity (body mass index $\geq 30$ ) had a relative risk of 3.10 (95\% confidence interval 2.69 to 3.57 ) of developing obesity, compared with children of mothers in the normal body weight range (body mass index 18.5-24.9). Offspring of women who were former or current smokers were also at a higher risk (1.16 (1.01 to 1.33) and 1.55 (1.25 to 1.92), respectively) of incident obesity than children of mothers who never smoked. By contrast, higher levels of physical activity and moderate alcohol intake were associated with a lower risk of offspring obesity. For example, the relative risks of obesity were 0.58 (0.46 to 0.74 ) in offspring of mothers who engaged in 150-299 minutes/week in exercise of moderate or vigorous intensity and 0.73 (0.57 to 0.94 ) in offspring of mothers who did more than 300 minutes/week, as compared with offspring of mothers who did not perform any exercise of moderate or vigorous intensity.

Compared with offspring of mothers who did not drink alcohol, light (1.0-4.9 g/day) and moderate (5.0$14.9 \mathrm{~g} /$ day) alcohol intake in mothers were associated with a reduced risk of obesity in offspring: relative risks were 0.89 (95\% confidence interval 0.78 to 1.01 ) and 0.80 (0.67 to 0.96), respectively. Alternate Healthy Eating Index 2010 diet score was not associated with the risk of offspring obesity. When we compared the top and bottom $20 \%$ groups of the Alternate Healthy Eating Index 2010 diet score, the relative risk was 0.99 (0.82 to 1.19).

Table 3 presents the offspring obesity risk based on each low risk category of maternal lifestyle. The risk of obesity was $56 \%$ (relative risk $0.44,95 \%$ confidence interval 0.39 to 0.50 ) lower in offspring of women with a healthy body weight (body mass index 18.5-24.9) than children of mothers in other body mass index categories (that is, body mass index $<18.5$ or $\geq 25.0$ ). Compared with offspring of women who were current smokers, children of non-smoking mothers had 31\% $(0.69,0.56$ to 0.86$)$ lower risk of obesity. Adherence to recommended levels of physical activity $(\geq 150$ $\mathrm{min} /$ week of moderate/vigorous exercise) and light to moderate intake of alcohol (1.0-14.9 g/day) in mothers 


\begin{tabular}{|c|c|}
\hline Characteristics & Value \\
\hline \multicolumn{2}{|l|}{ Maternal characteristics } \\
\hline No of mothers & 16945 \\
\hline Age at baseline (years; mean (SD)) & $41.3(4.3)$ \\
\hline White race or ethnicity (No (\%)) & $16398(96.8)$ \\
\hline Chronic diseases* (No (\%)) & $648(3.8)$ \\
\hline \multicolumn{2}{|l|}{ Lifestyle factors } \\
\hline Alternate Healthy Eating Index 2010 (diet score; mean (SD)) & $45.4(10.9)$ \\
\hline Body mass index (mean (SD)) & $25.1(5.2)$ \\
\hline Moderate/vigorous intensity exercise (min/week; median (IQR)) & $75.1(18.0-180.9)$ \\
\hline Alcohol intake (g/day; median (IQR)) & $1.2(0.0-4.7)$ \\
\hline \multicolumn{2}{|l|}{ Smoking status (No (\%)) } \\
\hline Never smoker & $11778(69.5)$ \\
\hline Former smoker & $4029(23.8)$ \\
\hline Current smoker & $1138(6.7)$ \\
\hline \multicolumn{2}{|l|}{ Pregnancy complications with offspring included in study (No (\%)) } \\
\hline Gestational diabetes & $517(3.1)$ \\
\hline Pre-eclampsia & $724(4.3)$ \\
\hline Pregnancy induced hypertension & $712(4.2)$ \\
\hline Cesarean delivery & $3281(19.4)$ \\
\hline Missing data & $2520(14.9)$ \\
\hline \multicolumn{2}{|l|}{ Social economic status (household income US\$; No (\%)) } \\
\hline$<\$ 50000$ & $1732(10.2)$ \\
\hline$\$ 50000$ to $\$ 99999$ & $6481(38.2)$ \\
\hline$\geq 100000$ & $5499(32.5)$ \\
\hline Missing data & $3233(19.1)$ \\
\hline Living with a spouse or partner (No (\%)) & $15452(91.2)$ \\
\hline \multicolumn{2}{|l|}{ Education of spouse/partner (No (\%)) } \\
\hline High school or less & $2612(15.4)$ \\
\hline College graduate & $7889(46.6)$ \\
\hline Graduate degree & $5104(30.1)$ \\
\hline Missing data & $1340(7.9)$ \\
\hline \multicolumn{2}{|l|}{ Offspring characteristics } \\
\hline No of children & 24289 \\
\hline Male sex (No (\%)) & $11133(45.8)$ \\
\hline Age at baseline (mean (SD)) & $12.1(1.9)$ \\
\hline Alternate Healthy Eating Index 2010 (diet score, mean (SD)) & $35.3(7.4)$ \\
\hline Physical activity (h/week, mean (SD)) & $16.2(10.8)$ \\
\hline Sedentary time (h/week, mean (SD)) & $46.8(23.2)$ \\
\hline Total energy intake (kcal/day) & $2152(701)$ \\
\hline Having breakfast $>5$ times/week (No (\%)) & $18126(74.6)$ \\
\hline Not consuming ready-made dinners at home (No (\%)) & $13821(56.9)$ \\
\hline Not consuming fried foods at home (No (\%)) & $16382(67.4)$ \\
\hline \multicolumn{2}{|l|}{ Birth weight group (kg; No (\%)) } \\
\hline$<2.30$ & $447(1.8)$ \\
\hline $2.30-3.19$ & $4042(16.6)$ \\
\hline $3.20-3.89$ & $10055(41.4)$ \\
\hline $3.90-4.49$ & $5049(20.8)$ \\
\hline$\geq 4.50$ & $493(2.0)$ \\
\hline Missing data & $4203(17.4)$ \\
\hline \multicolumn{2}{|l|}{ Gestational age at delivery (weeks; No (\%) ) } \\
\hline$<37$ & $1387(5.7)$ \\
\hline 37-39 & $4781(19.7)$ \\
\hline $40-42$ & $12881(53.0)$ \\
\hline$\geq 43$ & $1163(4.8)$ \\
\hline Missing data & $4077(16.8)$ \\
\hline
\end{tabular}

were both associated with a lower risk of offspring obesity (0.79 (0.69 to 0.91$)$ and 0.88 (0.79 to 0.99$)$, respectively). Offspring of women who were in top 40\% of the Alternate Healthy Eating Index 2010 diet score 


\begin{tabular}{|c|c|c|c|c|c|}
\hline \multirow[b]{2}{*}{ Maternal risk factor } & \multirow[b]{2}{*}{ No $(\%)$ of mothers } & \multirow[b]{2}{*}{ No (\%) of offspring } & \multirow[b]{2}{*}{ No (\%) of obese offspring } & \multicolumn{2}{|c|}{ Relative risk $(95 \% \mathrm{Cl})$} \\
\hline & & & & Model 1 & Model 2 \\
\hline \multicolumn{6}{|c|}{ Alternate Healthy Eating Index 2010 diet score } \\
\hline Fifth 1 (bottom) & $3380(20.0)$ & $4821(19.9)$ & $319(6.6)$ & 1.00 (reference) & 1.00 (reference) \\
\hline Fifth 2 & $3354(19.8)$ & 4835 (19.9) & $243(5.0)$ & 0.83 (0.70 to 0.99) & $0.84(0.71$ to 1.00$)$ \\
\hline Fifth 3 & $3390(20.0)$ & $4860(20.0)$ & $259(5.3)$ & $0.92(0.78$ to 1.09$)$ & $0.93(0.78$ to 1.10$)$ \\
\hline Fifth 4 & 3396 (20.0) & $4879(20.0)$ & $230(4.7)$ & 0.85 (0.70 to 1.05$)$ & $0.86(0.70$ to 1.05$)$ \\
\hline Fifth 5 (top) & $3425(20.2)$ & $4894(20.2)$ & $231(4.7)$ & $0.98(0.81$ to 1.17$)$ & $0.99(0.82$ to 1.19$)$ \\
\hline \multicolumn{6}{|l|}{ Body mass index } \\
\hline$<18.5$ & $209(1.2)$ & $307(1.2)$ & $2(0.7)$ & $0.23(0.06$ to 0.86$)$ & $0.23(0.06$ to 0.84$)$ \\
\hline $18.5-24.9$ & $9514(56.2)$ & $13731(56.9)$ & $444(3.2)$ & 1.00 (reference) & 1.00 (reference) \\
\hline 25.0-29.9 & $4437(26.2)$ & $6371(26.0)$ & $402(6.3)$ & $1.84(1.58$ to 2.14$)$ & $1.83(1.57$ to 2.13$)$ \\
\hline$\geq 30.0$ & $2785(16.4)$ & $3880(15.9)$ & $434(11.2)$ & $3.17(2.76$ to 3.64$)$ & $3.10(2.69$ to 3.57$)$ \\
\hline \multicolumn{6}{|c|}{ Moderate/vigorous intensity exercise (min/week) } \\
\hline 0 & $1009(6.0)$ & $1320(5.4)$ & $120(9.1)$ & 1.00 (reference) & 1.00 (reference) \\
\hline $1-149$ & $10096(59.6)$ & $14430(59.4)$ & $824(5.7)$ & $0.73(0.61$ to 0.88$)$ & $0.74(0.61$ to 0.89$)$ \\
\hline 150-299 & $3659(21.6)$ & $5293(21.8)$ & $197(3.7)$ & $0.58(0.46$ to 0.73$)$ & $0.58(0.46$ to 0.74$)$ \\
\hline$\geq 300$ & $2181(12.8)$ & $3246(13.4)$ & $141(4.3)$ & $0.72(0.56$ to 0.92$)$ & $0.73(0.57$ to 0.94$)$ \\
\hline \multicolumn{6}{|l|}{ Smoking status } \\
\hline Never smoker & $11737(69.3)$ & $17068(70.3)$ & $847(5.0)$ & 1.00 (reference) & 1.00 (reference) \\
\hline Former smoker & $4197(24.7)$ & $5916(24.3)$ & $332(5.6)$ & $1.18(1.03$ to 1.35$)$ & $1.16(1.01$ to 1.33$)$ \\
\hline Current smoker & $1011(6.0)$ & $1305(5.4)$ & $103(7.9)$ & $1.62(1.30$ to 2.02$)$ & $1.55(1.25$ to 1.92$)$ \\
\hline \multicolumn{6}{|l|}{ Alcohol intake (g/day) } \\
\hline 0 & $5492(32.4)$ & $7842(32.3)$ & $508(6.5)$ & 1.00 (reference) & 1.00 (reference) \\
\hline $1.0-4.9$ & $7194(42.5)$ & $10219(42.1)$ & $525(5.1)$ & 0.88 (0.77 to 0.99$)$ & $0.89(0.78$ to 1.01$)$ \\
\hline $5.0-14.9$ & $3253(19.2)$ & 4704 (19.4) & $194(4.1)$ & $0.78(0.66$ to 0.94$)$ & $0.80(0.67$ to 0.96$)$ \\
\hline$\geq 15.0$ & $1006(5.9)$ & $1524(6.2)$ & $55(3.6)$ & 0.68 (0.49 to 0.95$)$ & $0.70(0.50$ to 0.97$)$ \\
\hline
\end{tabular}

Model 1 adjusted for mother's age at baseline (in 5-year categories), offspring sex (boy/girl), and other lifestyle factors included in the table; model 2 additionally adjusted for race/ethnicity (white, yes/no), chronic diseases (prevalent diabetes, cardiovascular disease, or cancer), living status (with spouse/partner or not), household income ( $<$ US $\$ 50000$, \$50 000-\$99999,

$\geq \$ 100000$; US $\$ 1=£ 0.75, € 0.85$ ), and educational attainment of spouse/partner (high school, college, graduate school).

did not have a significantly lower risk of obesity (0.97, 0.83 to 1.12 ) than children of mothers who were in the bottom $60 \%$ of the diet score.

We also evaluated combinations of maternal low risk lifestyle factors in relation to offspring obesity. An increasing number of low risk factors was significantly associated with a monotonically lower risk of offspring obesity (table 4, model 1) after controlling for maternal covariates. Compared with children of mothers who did not adhere to any low risk factor, offspring of women who adhered to three low risk factors (healthy diet, $\geq 150 \mathrm{~min} /$ week of moderate/vigorous physical activity, and light to moderate alcohol consumption) had a 23\% lower risk (relative risk $0.77,95 \%$ confidence interval 0.59 to 1.00) of obesity, independently of maternal body mass index and smoking status. Further addition of smoking status to the combination of low risk factors was associated with a $42 \%(0.58,0.36$ to 0.93$)$ lower risk of offspring obesity, independently of maternal levels of body mass index. Lastly, children of mothers who adhered to all five low risk factors had 75\% lower risk $(0.25,0.14$ to 0.47$)$ of obesity, compared with offspring of women who did meet any of the low risk factors.

The associations between maternal lifestyle and childhood obesity were not substantially different in analyses stratified by sex and age (fig 1 and supplementary table 1), or by potential effect modifiers, including pregnancy complications, birth weight, gestational age, and gestational weight gain (supplementary table 2). Similarly, in analyses restricted within mothers without missing covariate

\begin{tabular}{|c|c|c|c|c|c|}
\hline \multirow[b]{2}{*}{ Maternal low risk lifestyle factors } & \multirow[b]{2}{*}{ No (\%) of mothers } & \multirow[b]{2}{*}{ No (\%) of offspring } & \multirow[b]{2}{*}{ No (\%) of obese offspring } & \multicolumn{2}{|c|}{ Relative risk $(95 \% \mathrm{Cl})$} \\
\hline & & & & Model 1 & Model 2 \\
\hline $\begin{array}{l}\text { Healthy diet (based on the Alternate Healthy } \\
\text { Eating Index } 2010 \text { diet score) }\end{array}$ & $6821(40.3)$ & $9773(40.2)$ & $461(4.7)$ & 0.96 (0.83 to 1.11$)$ & 0.97 (0.83 to 1.12$)$ \\
\hline Body mass index 18.5-24.9 & $9514(56.4)$ & $13731(56.5)$ & $444(3.2)$ & $0.43(0.38$ to 0.49$)$ & $0.44(0.39$ to 0.50$)$ \\
\hline Moderate/vigorous exercise $\geq 150 \mathrm{~min} /$ week & $5840(34.5)$ & $8539(35.2)$ & $338(4.0)$ & 0.78 (0.68 to 0.90$)$ & 0.79 (0.69 to 0.91$)$ \\
\hline Current non-smoker & $15934(94.0)$ & $22984(94.6)$ & $1179(5.1)$ & $0.66(0.53$ to 0.81$)$ & $0.69(0.56$ to 0.86$)$ \\
\hline Alcohol intake (1.0-14.9 g/day) & $10447(61.7)$ & $14923(61.4)$ & $719(4.8)$ & 0.87 (0.77 to 0.97$)$ & $0.88(0.79$ to 0.99$)$ \\
\hline
\end{tabular}




\begin{tabular}{|c|c|c|c|c|c|}
\hline \multirow[b]{2}{*}{ No of low risk factors } & \multirow[b]{2}{*}{ No (\%) of mothers } & \multirow[b]{2}{*}{ No (\%) of offspring } & \multirow[b]{2}{*}{ No (\%) of obese offspring } & \multicolumn{2}{|c|}{ Relative risk $(95 \% \mathrm{Cl})$} \\
\hline & & & & Model 1 & Model 2 \\
\hline \multicolumn{6}{|c|}{$\begin{array}{l}\text { Three low-risk factors-healthy diet (top 40\% of Alternate Healthy Eating Index } 2010 \text { diet score), moderate/vigorous exercise } \geq 150 \text { min/week, light to moderate alcohol intak } \\
(1.0-14.9 \mathrm{~g} / \text { day) }\end{array}$} \\
\hline 0 & $3141(18.5)$ & $4452(18.3)$ & $306(6.9)$ & 1 (reference) & 1 (reference) \\
\hline 1 & $6584(38.8)$ & 9461 (38.9) & $547(5.7)$ & 0.91 (0.77 to 1.06$)$ & 0.90 (0.78 to 1.05$)$ \\
\hline 2 & $5136(30.3)$ & $7354(30.2)$ & $316(4.3)$ & 0.79 (0.67 to 0.94) & 0.81 (0.68 to 0.96$)$ \\
\hline 3 & $2084(12.3)$ & $3022(12.4)$ & $113(3.7)$ & 0.77 (0.59 to 1.00$)$ & 0.77 (0.59 to 1.01) \\
\hline \multicolumn{6}{|c|}{ Four low risk factors-above three factors plus non-smoking } \\
\hline 0 & $212(1.3)$ & $270(1.1)$ & $23(8.5)$ & 1 (reference) & 1 (reference) \\
\hline 1 & $3410(20.1)$ & $4825(19.8)$ & $337(7.0)$ & $0.78(0.52$ to 1.18$)$ & 0.76 (0.51 to 1.13$)$ \\
\hline 2 & $6340(37.4)$ & $9108(37.5)$ & $513(5.6)$ & $0.68(0.45$ to 1.04$)$ & 0.67 (0.45 to 1.01$)$ \\
\hline 3 & $4980(29.3)$ & $7166(29.5)$ & $302(4.2)$ & $0.60(0.39$ to 0.91$)$ & 0.59 (0.40 to 0.89$)$ \\
\hline 4 & $2003(11.8)$ & $2920(12.0)$ & $107(3.7)$ & 0.58 (0.36 to 0.93$)$ & 0.58 (0.37 to 0.91$)$ \\
\hline \multicolumn{6}{|c|}{ Five low-risk factors-above four factors plus healthy weight (body mass index 18.5-24.9) } \\
\hline 0 & $117(0.7)$ & $145(0.6)$ & $15(10.3)$ & 1 (reference) & 1 (reference) \\
\hline 1 & $1912(11.2)$ & $2702(11.1)$ & $246(9.1)$ & $0.83(0.49$ to 1.43$)$ & $0.80(0.48$ to 1.35$)$ \\
\hline 2 & $4541(26.8)$ & $6455(26.5)$ & $446(6.9)$ & $0.63(0.37$ to 1.09$)$ & $0.62(0.37$ to 1.05$)$ \\
\hline 3 & $5332(31.4)$ & $7641(31.4)$ & $348(4.6)$ & $0.43(0.25$ to 0.73$)$ & $0.44(0.26$ to 0.74$)$ \\
\hline 4 & $3649(21.5)$ & $5315(21.9)$ & $176(3.3)$ & 0.32 (0.19 to 0.55$)$ & $0.34(0.20$ to 0.58$)$ \\
\hline 5 & $1394(8.2)$ & $2031(8.4)$ & $51(2.5)$ & 0.25 (0.14 to 0.47$)$ & 0.27 (0.15 to 0.49$)$ \\
\hline
\end{tabular}

values or among offspring without imputed body mass index assessments, the results did not change materially (supplementary tables 3 and 4, respectively). The risk of obesity in offspring of mothers who adhered to all five low risk factors did not change materially when former smokers were removed from the low risk group (supplementary table 5). Lastly, when we adjusted for prenatal maternal lifestyle factors, we observed largely similar associations (supplementary table 6).

We further explored the role of offspring lifestyle factors in the associations between maternal lifestyle and childhood obesity risk. Supplementary figure 3 shows the correlation coefficients between maternal lifestyle and offspring lifestyle. Maternal dietary and lifestyle factors were weakly to modestly correlated with corresponding offspring factors. Of all lifestyle factors, diet and body mass index showed stronger correlations between mothers and their children; the Spearman correlation coefficients were 0.37 and 0.28 , respectively. Further adjustment of offspring lifestyle did not materially alter the association between maternal lifestyle and childhood obesity (relative risk 0.27 (95\% confidence interval 0.15 to 0.49 ); table 4, model 2).

Lastly, table 5 shows the risk of obesity in offspring according to the combination of lifestyle factors of mothers and their offspring. The combination of low risk maternal and offspring lifestyles was associated with an $82 \%$ (relative risk 0.18, 95\% confidence interval 0.09 to 0.37 ) lower risk of offspring obesity than the combination of high risk maternal and offspring lifestyles.

Adjusted for mother's age at baseline (in 5-year categories), race/ethnicity (white, others), chronic diseases (prevalence of diabetes, cardiovascular disease, or cancer), living status (with spouse/ partner or not), household income (<US\$50000, $\$ 50$ 000-\$99 999, $\geq \$ 100000 ;$ US $\$ 1=€ 0.75$, €0.85), and educational attainment of spouse/partner (high school, college, graduate school); and offspring factors, including sex (boy, girl) and calories intake (kcal/day, categorized in tenths). Mothers in the study were categorized into low risk and high risk groups, based on the number of low risk factors they adhered (low risk=women who adhered to 3-5 low risk lifestyle factors; high risk=women who adhered to 0-2 low risk lifestyle factors. In offspring, the low risk and high risk categories were defined for each lifestyle factor (low risk=top 40\% of Alternate Healthy Eating Index 2010 diet score; top third for physical activities; bottom third of sedentary time; and eating breakfast every day of the week and not eating ready-made dinners or fried foods at home or elsewhere for dining behaviors; high risk=remainder of each offspring lifestyle factor).

\section{Discussion}

An overall healthy lifestyle of mothers during their offspring's childhood and adolescence was associated with a substantially lower risk of developing obesity in their children. Offspring of women who adhered to five low risk lifestyle factors (a high quality diet, normal body weight, regular physical activities, light to moderate intake of alcohol, and non-smoking) had a 75\% lower risk of developing incident obesity than children of mothers who did not adhere to any of the low risk lifestyle factors. The risk of incident offspring obesity was $82 \%$ lower when both mothers and their offspring followed a healthy lifestyle. These associations were independent of other established and potential risk factors of childhood 

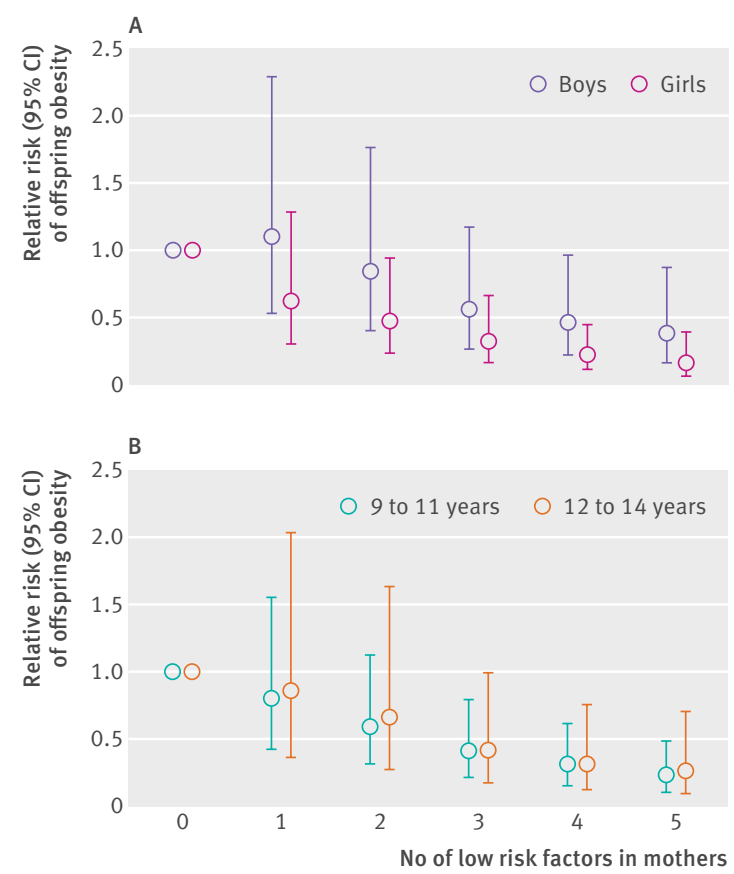

Fig 1 | Associations between maternal low risk lifestyle factors and risk of offspring obesity, stratified by (A) sex and (B) age. Multivariable analyses adjusted for mother's age at baseline (in 5-year categories), race/ethnicity (white, others), chronic diseases (prevalence of diabetes, cardiovascular disease or cancer), living status (with spouse/partner or not), household income ( $U$ US $\$ 50000$, $\$ 50000-\$ 99999, \geq \$ 100000 ;$ US $\$ 1=f 0.75, € 0.85)$, and educational attainment of spouse/partner (high school, college, graduate school); and offspring sex (boy, girl) for the analysis in figure $1 \mathrm{~B}$.

obesity and persisted among participants who had different baseline risk profiles defined by pregnancy complications and other maternal factors.

\section{Comparison with other studies}

Childhood obesity research has documented that the development of obesity could result from both genetic and environmental factors, as well as their interactions. $^{36}$ Studies of twins have suggested a strong genetic underpinning for obesity, ${ }^{5}$ although rising prevalence of obesity during past decades has indicated the critical role of environmental factors in c. ${ }^{6-9}$ Recently, research has started focusing on the home environment of children by evaluating the lifestyle factors of mothers during offspring childhood and adolescence and their children's obesity risk. For example, studies have shown that the risk of offspring obesity doubled when mothers were obese during their children's childhood and adolescence. ${ }^{8}$ Moreover, maternal physical activity and dietary intake influence offspring levels of exercise and food choices. ${ }^{13-16}$ Children of physically active mothers are more likely to be active than children of inactive mothers. ${ }^{13} 14$ Likewise, research has reported similarities between the dietary practices of children and their care givers. ${ }^{15}$ 16

In addition, maternal behaviors such as smoking and alcohol drinking were also associated with body mass index in the offspring. ${ }^{17} 18$ A large multicenter study composed of 77192 children and 194727 adolescents documented that body mass index of children exposed to maternal smoking was 0.08 greater than those who were not. ${ }^{17}$ A recent study conducted in Finland showed that adolescents of mothers who consumed alcohol in moderation (score 1-4 according to the Alcohol Use Disorders Identification $\mathrm{Test}^{37}$ ) had a lower risk of being underweight than adolescents of abstainer mothers. ${ }^{18}$ Despite this existing knowledge base, no study has examined the overall effect of a healthy maternal lifestyle on the risk of offspring obesity.

In the current study, we extended the knowledge beyond the role of individual maternal lifestyle factors to the effect of overall maternal lifestyle during the offspring's childhood and adolescence with the risk of offspring obesity. In line with previous studies, we found that maternal obesity, smoking, and physical inactivity were strongly associated with offspring obesity. ${ }^{8} 13$ 1417 Although the literature is limited regarding the association of maternal alcohol consumption on offspring obesity, ${ }^{18}$ we found that offspring of mothers who consumed alcohol no more than moderation had a lower risk of obesity than offspring of mothers who abstained. In our NHSII participants, few women drank heavily $(0.25 \%$ reported drinking more than 45 $\mathrm{g}$ of alcohol per day). Thus, our findings only pertain to low to moderate drinking of alcohol and cannot be further extrapolated to heavy use of alcohol intake. Unexpectedly, in our study, maternal dietary pattern during offspring's childhood and adolescence was not associated with offspring obesity. Evidence from existing studies did not generally support a consistent association between most dietary factors and obesity risk among children. ${ }^{38}$ One potential explanation for our observation of a null association between maternal diet and childhood obesity is that children's energy intake might not be exclusively from meals prepared at home because children's diet is influenced by multiple factors including school and neighborhood food environments and peer influences. Indeed, a nationally representative study of energy intake trend among US children by eating location showed that the percentage of calories eaten away from home increased from $23.4 \%$ to $33.9 \%$ from 1977 to $2006 .{ }^{39}$ In addition, under-reporting of dietary intake among children can be a serious concern in childhood obesity studies, because children with overweight and obesity are more prone to under-report food intake. ${ }^{4041} \mathrm{~A}$ recent meta-analysis among children aged 4-16 years concluded that there was only a modest correlation ( $r=0.48$ for total energy intake) between self reported diet and diet assessed by objective instruments, including biomarkers or direct observation of food intake by trained researchers. ${ }^{42}$ The observed modest 


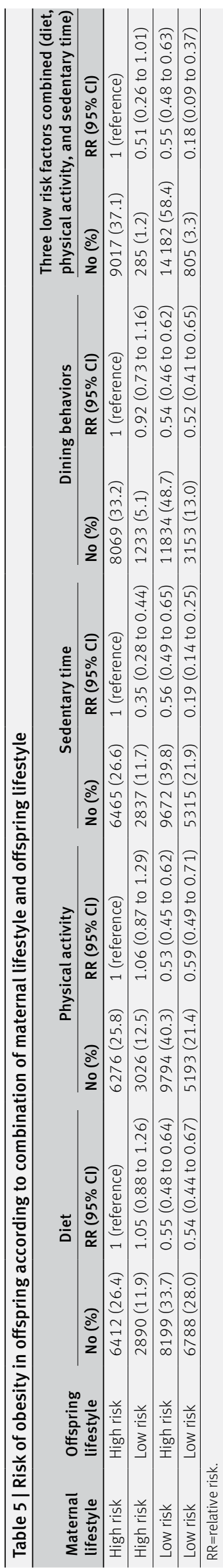

correlation between children's diet and mother's diet in the current study might at least partly reflect these methodological challenges.

In a recent analysis, we showed the importance of an overall healthy lifestyle before pregnancy as a modifiable factor for the prevention of obesity in the next generations. ${ }^{43}$ In the current study, we provided new evidence suggesting that maternal lifestyle during their offspring's childhood was also critical and might influence children's obesity risk independently of prenatal maternal lifestyle. These findings highlight the crucial role of maternal lifestyle at early life stages of children in their risk of developing obesity.

\section{Mechanisms and clinical implications}

The mechanisms underlying our observations are not completely understood, although the effect of mothers' lifestyle and behaviors could exert a critical impact on their children's lifestyle and diet and subsequently modulate offspring's obesity risk. ${ }^{13-16}$ Regarding physical activity, the possible explanations for the effect of maternal physical activity on their children's weight includes increasing activity levels in children by encouragement for engaging in activities and sports, role modeling, and shared activities. ${ }^{13} 14$ Moore and colleagues showed that children of active mothers had a twofold increase in activities compared with offspring of inactive women. ${ }^{13}$

The mechanism by which maternal smoking behavior and moderate alcohol consumption affect offspring weight is unclear. Second hand smoking could be the mechanism underlying the link between maternal smoking and offspring obesity. ${ }^{19}$ Alternatively, maternal smoking behavior could reflect an unhealthy family lifestyle in general. The association between these behaviors and mental wellbeing could also explain our results. Smoking in adults is associated with an increased risk of depression, ${ }^{44}$ whereas moderate alcohol intake is related to decreased anxiety and depression. ${ }^{45}$ Research suggests that maternal depression is associated with an increased body mass index in offspring. ${ }^{46}$ Future studies are needed to further understand mechanisms explaining how behaviors such as smoking and drinking affect body weight in offspring during childhood and adolescence.

Our study showed that the impact of mothers' lifestyle on offspring obesity outweighed that of offspring's lifestyle. Several factors could contribute to the weak mediation effect of children's lifestyle in the association between maternal lifestyle and childhood obesity risk. One of the most plausible reasons is that not all aspects of child lifestyle were captured in our research. For instance, we did not assess the children's exposure to second hand smoking, which is an established risk factor for children's health. ${ }^{4748}$ Measurement errors in children's lifestyle assessments might also have a role. Research has shown that children with obesity tend to under-report their energy intake ${ }^{4041}$ and over-report their total amount of physical activities. ${ }^{49}$ Although the self reported lifestyle practices have been extensively validated in the NHS, 
we have not examined the accuracy of children's self reported lifestyle assessments.

Overall, these methodological limitations could partly explain the weak influence of offspring lifestyle on the associations between maternal lifestyle and offspring obesity. Moreover, the etiology of childhood obesity is complicated and could involve a network of diet, behaviors, psychosocial determinants, and other factors. ${ }^{50}$ It is likely that mothers' lifestyle might exert effects on childhood obesity risk through pathways other than children's lifestyle, although more research is needed in this regard. Nonetheless, our findings are in line with a recent large scale clinical study showing that parent-only interventions were as effective as family based interventions to promote weight loss in children, ${ }^{5152}$ highlighting the role of parental behaviors in the etiology of offspring's obesity risk.

\section{Strengths and limitations of study}

Strengths of our study included a large sample size and a prospective study design with long follow-up, which allowed us to examine the impact of maternal factors before the occurrence of offspring obesity in childhood and adolescence. The detailed information of lifestyle factors in both mothers and offspring enabled us to conduct comprehensive analyses by taking into account many factors, including offspring behaviors, such as dietary habits, physical activity and sedentary time, and calorie intake.

However, several limitations should be considered. Firstly, the lifestyle characteristics, including body weight, of mothers and their offspring were exclusively based on self reports, which are known to be subject to measurement errors. However, the accuracy of self reported body weight has been examined in a validation study among a random subset of NHSII participants living in the Boston area, and their self reported weight were found to be highly reliable. ${ }^{53}$ Similar validation studies in children have also indicated reasonable accuracy of self reported weight and height. ${ }^{30-33}$ For example, in the nationally representative National Longitudinal Study of Adolescent Health, researchers have shown that body mass index based on self reported height and weight correctly classified 96\% children in terms of their obesity status. ${ }^{31}$

Secondly, our NHSII participants had a relatively homogeneous socioeconomic status and educational attainment. In addition, the obesity prevalence in offspring was lower in our GUTS participants than in other populations in the USA. These characteristics could limit the generalizability of our findings to populations with different socioeconomic status or health status. Thirdly, a loss to follow-up is a concern for the current study, although our propensity score analysis showed that the probability of loss to followup was independent of maternal lifestyle.

Fourthly, we imputed missing values of body mass index in offspring across the follow-up. We examined the validity of imputed values by comparing valid body mass index assessments with imputed values in classifying the obesity status of GUTS participants. We found an excellent specificity but a modest sensitivity of the imputed values in correctly classifying the obesity status defined by valid body mass index assessments. Moreover, all offspring included in our study had at least one valid measure of body mass index, and 90\% of them had two or more valid values of body mass index. Therefore, the impact of this misclassification was limited.

Another limitation, as in any observational study, is that we cannot exclude the possibility of uncontrolled confounding by psychosocial factors in offspring or residual confounding. Lastly, our study only examined maternal lifestyle, and the potentially crucial role of paternal lifestyle in the development of obesity in offspring was not investigated. Prospective research examining father's role in the development of obesity in offspring is needed.

\section{Conclusion}

Our study shows that mothers' overall healthy lifestyle during the period of their offspring's childhood and adolescence is associated with a substantially lower risk of obesity in their children. Importantly, adherence to a healthy lifestyle in both mothers and their children could result in an even further reduction in the risk of offspring obesity. Our findings highlight the potentially critical role of maternal lifestyle choices in the etiology of childhood obesity and lend support to family or parent based intervention strategies for reducing childhood obesity risk.

Contributors: KD and QS had full access to all of the data in the study and takes responsibility for the integrity of the data and the accuracy of the data analysis. KD and QS planned and designed the study, and analyzed and interpreted the data. KD drafted the manuscript. All authors contributed to the interpretation of the results and critical revision of the manuscript for important intellectual content and approved the final version of the manuscript. KD and QS are the guarantors of this investigation. The corresponding author attests that all listed authors meet authorship criteria and that no others meeting the criteria have been omitted.

Funding: This study was supported by grants UM1-CA176726, P30-DK046200, U54-CA155626, T32-DK007703-16, HD066963, HL096905, DK084001, OH009803, and MH087786 from the US National Institutes of Health. QS is supported by grants ES021372, ES022981, and HL34594 from the National Institutes of Health.

Competing interests: All authors have completed the ICMJE uniform disclosure form at www.icmje.org/coi_disclosure.pdf and declare: support from the National Institutes of Health for the submitted work; no financial relationships with any organizations that might have an interest in the submitted work in the previous three years; no other relationships or activities that could appear to have influenced the submitted work.

Ethical approval: The Nurses' Health Study II and Growing Up Today Study were approved by the institutional review board at Brigham and Women's Hospital and Harvard TH Chan School of Public Health. The return of the completed self administrated questionnaire was considered to imply informed consent.

Data sharing: No additional data available.

The lead authors affirm that the manuscript is an honest, accurate, and transparent account of the study being reported; that no important aspects of the study have been omitted; and that any discrepancies from the study as planned have been explained.

This is an Open Access article distributed in accordance with the Creative Commons Attribution Non Commercial (CC BY-NC 4.0) license, which permits others to distribute, remix, adapt, build upon this work non-commercially, and license their derivative works on different terms, provided the original work is properly cited and the use is noncommercial. See: http://creativecommons.org/licenses/by-nc/4.0/. 
1 Ogden CL, Carroll MD, Lawman HG, et al. Trends in obesity prevalence among children and adolescents in the United States, 1988-1994 through 2013-2014. JAMA 2016;315:2292-9. doi:10.1001/ jama.2016.6361

2 Freedman DS, Dietz WH, Srinivasan SR, Berenson GS. The relation of overweight to cardiovascular risk factors among children and adolescents: the Bogalusa Heart Study. Pediatrics 1999;103:117582. doi:10.1542/peds.103.6.1175

3 Flegal KM, Kit BK, Orpana H, Graubard BI. Association of all-cause mortality with overweight and obesity using standard body mass index categories: a systematic review and meta-analysis. IAMA 2013.309.71-82 doi:10.1001/jama.2012.113905

4 Di Angelantonio E, Bhupathiraju ShN, Wormser D, et al, Global BM Mortality Collaboration. Body-mass index and all-cause mortality: individual-participant-data meta-analysis of 239 prospective studies in four continents. Lancet 2016;388:776-86. doi:10.1016/S01406736(16)30175-1

5 Maes HH, Neale MC, Eaves LJ. Genetic and environmental factors in relative body weight and human adiposity. Behav Genet 1997;27:325-51. doi:10.1023/A:1025635913927

6 Dubois L, Girard M. Early determinants of overweight at 4.5 years in a population-based longitudinal study. Int J Obes (Lond) 2006;30:6107. doi:10.1038/sj.ijo.0803141

7 Faith MS, Heshka S, Keller KL, et al. Maternal-child feeding patterns and child body weight: findings from a population-based sample. Arch Pediatr Adolesc Med 2003;157:926-32. doi:10.1001/ archpedi.157.9.926

8 Whitaker RC, Wright JA, Pepe MS, Seidel KD, Dietz WH. Predicting obesity in young adulthood from childhood and parental obesity. N Engl / Med 1997;337:869-73. doi:10.1056/ NEJM199709253371301

9 Anderson PM, Butcher KF, Schanzenbach DW. Childhood disadvantage and obesity: Is nurture trumping nature? National Bureau of Economic Research, 2007. doi:10.3386/w13479

10 Davison KK, Birch LL. Childhood overweight: a contextual model and recommendations for future research. Obes Rev 2001;2:159-71. doi:10.1046/j.1467-789x.2001.00036.x

11 Steinbeck KS. The importance of physical activity in the prevention of overweight and obesity in childhood: a review and an opinion. Obes $\operatorname{Rev} 2001 ; 2: 117-30$. doi:10.1046/j.1467-789x.2001.00033.x

12 Klesges RC, Klesges LM, Eck LH, Shelton ML. A longitudinal analysis of accelerated weight gain in preschool children. Pediatrics 1995;95:126-30.

13 Moore LL, Lombardi DA, White MJ, Campbell JL, Oliveria SA, Ellison RC. Influence of parents' physical activity levels on activity levels of young children. J Pediatr 1991;118:215-9. doi:10.1016/S00223476(05)80485-8

14 Gustafson SL, Rhodes RE. Parental correlates of physical activity in children and early adolescents. Sports Med 2006;36:79-97. doi:10.2165/00007256-200636010-00006

15 Acharya K, Feese M, Franklin F, Kabagambe EK. Body mass index and dietary intake among Head Start children and caregivers. J Am Diet Assoc 2011;111:1314-21. doi:10.1016/j.jada.2011.06.013

16 Stang J, Loth KA. Parenting style and child feeding practices: potential mitigating factors in the etiology of childhood obesity. J Am Diet Assoc 2011:111:1301-5. doi:10.1016/j.jada.2011.06.010

17 Braithwaite I, Stewart AW, Hancox RJ, Beasley R, Murphy R, Mitchell EA, ISAAC Phase Three Study Group. Maternal post-natal tobacco use and current parental tobacco use is associated with higher body mass index in children and adolescents: an international cross-sectional study. BMC Pediatr 2015;15:220. doi:10.1186/s12887-015-0538-x

18 Figueiredo RAO, Roos E, Eriksson JG, Simola-Ström S, Weiderpass E. Maternal alcohol and tobacco consumption and the association with their 9 to 14-year-old children's body mass index. Scand J Public Health 2017;45:503-10. doi:10.1177/1403494817702264

19 Raum E, Küpper-Nybelen J, Lamerz A, Hebebrand I, HerpertzDahlmann B, Brenner H. Tobacco smoke exposure before, during, and after pregnancy and risk of overweight at age 6. Obesity (Silver Spring) 2011;19:2411-7. doi:10.1038/oby.2011.129

20 Dawson IG, Dohle S. Towards an understanding of adult judgments of synergistic health benefits. BrJ Health Psychol 2016;21:204-23. doi:10.1111/bjhp.12158

21 Hu FB, Manson JE, Stampfer MJ, et al. Diet, lifestyle, and the risk of type 2 diabetes mellitus in women. N Engl J Med 2001;345:790-7. doi:10.1056/NEJMoa010492

22 Stampfer MJ, Hu FB, Manson JE, Rimm EB, Willett WC. Primary prevention of coronary heart disease in women through diet and lifestyle. N Engl J Med 2000;343:16-22. doi:10.1056/ NEJM200007063430103

23 van Dam RM, Li T, Spiegelman D, Franco OH, Hu FB. Combined impact of lifestyle factors on mortality: prospective cohort study in US women. BM/ 2008;337:a1440. doi:10.1136/bmj.a1440

24 Colditz GA, Hankinson SE. The Nurses' Health Study: lifestyle and health among women. Nat Rev Cancer 2005;5:388-96. doi:10.1038/nrc1608
25 Salvini S, Hunter DJ, Sampson L, et al. Food-based validation of a dietary questionnaire: the effects of week-to-week variation in food consumption. Int J Epidemiol 1989;18:858-67. doi:10.1093/ ije/18.4.858

26 Willett WC, Sampson L, Browne ML, et al. The use of a selfadministered questionnaire to assess diet four years in the past. Am J Epidemiol 1988;127:188-99. doi:10.1093/oxfordjournals.aje. a114780

27 Chiuve SE, Fung TT, Rimm EB, et al. Alternative dietary indices both strongly predict risk of chronic disease. J Nutr 2012;142:1009-18. doi:10.3945/jn.111.157222

28 Wolf AM, Hunter DJ, Colditz GA, et al. Reproducibility and validity of a self-administered physical activity questionnaire. Int J Epidemiol 1994;23:991-9. doi:10.1093/ije/23.5.991

29 Rockett HR, Breitenbach M, Frazier AL, et al. Validation of a youth/ adolescent food frequency questionnaire. Prev Med 1997;26:808 16. doi:10.1006/pmed.1997.0200

30 Strauss RS. Comparison of measured and self-reported weight and height in a cross-sectional sample of young adolescents. Int J Obes Relat Metab Disord 1999;23:904-8. doi:10.1038/sj.ijo.0800971

31 Goodman E, Hinden BR, Khandelwal S. Accuracy of teen and parental reports of obesity and body mass index. Pediatrics 2000;106:52-8. doi:10.1542/peds.106.1.52

32 Field AE, Aneja P, Rosner B. The validity of self-reported weight change among adolescents and young adults. Obesity (Silver Spring) 2007:15:2357-64. doi:10.1038/oby.2007.279

33 Himes JH, Faricy A. Validity and reliability of self-reported stature and weight of US adolescents. Am J Hum Biol 2001;13:25560. doi:10.1002/1520-6300(200102/03)13:2<255::AIDAJHB1036>3.0.CO;2-E

34 Cole TJ, Bellizzi MC, Flegal KM, Dietz WH. Establishing a standard definition for child overweight and obesity worldwide: international survey. BMJ 2000;320:1240-3. doi:10.1136/ bmj.320.7244.1240

35 Bland JM, Altman DG. Statistical methods for assessing agreement between two methods of clinical measurement. Lancet 1986;1:30710. doi:10.1016/S0140-6736(86)90837-8

36 Hebebrand J, Hinney A. Environmental and genetic risk factors in obesity. Child Adolesc Psychiatr Clin N Am 2009;18:83-94. doi:10.1016/j.chc.2008.07.006

37 Babor TF, Higgins-Biddle JC, Saunders JB, Monteiro MG. Audit: The alcohol use disorders identification test: Guidelines for use in primary health care. WHO, 2001. http://apps.who.int/iris/ handle/10665/67205

38 Newby PK. Are dietary intakes and eating behaviors related to childhood obesity? A comprehensive review of the evidence. J Law Med Ethics 2007;35:35-60. doi:10.1111/j.1748720X.2007.00112.x

39 Poti JM, Popkin BM. Trends in energy intake among US children by eating location and food source, 1977-2006. J Am Diet Assoc 2011;111:1156-64. doi:10.1016/j.jada.2011.05.007

40 Livingstone MB, Robson PJ. Measurement of dietary intake in children. Proc Nutr Soc 2000;59:279-93. doi:10.1017/ S0029665100000318

41 Maffeis C, Schutz Y, Zaffanello M, Piccoli R, Pinelli L. Elevated energy expenditure and reduced energy intake in obese prepubertal children: paradox of poor dietary reliability in obesity?] Pediatr 1994;124:348-54. doi:10.1016/S0022-3476(94)70355-8

42 Merson B, Pezdek K, Saywitz K. A meta-analysis of children's selfreports of dietary intake. Psychol Health 2017;32:186-203. doi:10.1 080/08870446.2016.1250274

43 Dhana K, Zong G, Yuan C, et al. Lifestyle of women before pregnancy and the risk of offspring obesity during childhood through early adulthood. Int J Obes (Lond) 2018. doi:10.1038/s41366-0180052-y

44 Bakhshaie J, Zvolensky MJ, Goodwin RD. Cigarette smoking and the onset and persistence of depression among adults in the United States: 1994-2005. Compr Psychiatry 2015;60:142-8. doi:10.1016/i.comppsych.2014.10.012

45 Bellos S, Skapinakis P, Rai D, et al. Longitudinal association between different levels of alcohol consumption and a new onset of depression and generalized anxiety disorder: Results from an international study in primary care. Psychiatry Res 2016;243:30-4 doi:10.1016/j.psychres.2016.05.049

46 Duarte CS, Shen S, Wu P, Must A. Maternal depression and child BMI: longitudinal findings from a US sample. Pediatr Obes 2012;7:12433. doi:10.1111/j.2047-6310.2011.00012.x

47 Weitzman M, Cook S, Auinger P, et al. Tobacco smoke exposure is associated with the metabolic syndrome in adolescents. Circulation 2005;112:862-9. doi:10.1161/ CIRCULATIONAHA.104.520650

48 Davis CL, Tingen MS, Jia J, et al. Passive Smoke Exposure and Its Effects on Cognition, Sleep, and Health Outcomes in Overweight and Obese Children. Child Obes 2016;12:119-25. doi:10.1089/ chi. 2015.0083 
49 Elliott SA, Baxter KA, Davies PS, Truby H. Accuracy of selfreported physical activity levels in obese adolescents. / Nutr Metab 2014:2014:808659. doi:10.1155/2014/808659

50 Blazer DG, Hernandez LM (eds). Genes, behavior, and the social environment: Moving beyond the nature/nurture debate. National Academies Press. 2006.

51 Boutelle KN, Rhee KE, Liang J, et al. Effect of Attendance of the Child on Body Weight, Energy Intake, and Physical Activity in Childhood Obesity Treatment: A Randomized Clinical Trial. JAMA Pediatr 2017;171:622-8. doi:10.1001/jamapediatrics.2017.0651
52 Ewald H, Kirby J, Rees K, Robertson W. Parent-only interventions in the treatment of childhood obesity: a systematic review of randomized controlled trials. J Public Health (Oxf) 2014;36:476-89. doi:10.1093/pubmed/fdt108

53 Rimm EB, Stampfer MJ, Colditz GA, Chute CG, Litin LB, Willett WC. Validity of self-reported waist and hip circumferences in men and women. Epidemiology 1990;1:466-73. doi:10.1097/00001648199011000-00009

Web appendix: Supplementary material 